\title{
PREVENCIÓN DE LA VIOLENCIA Y GÉNERO EN GUANAJUATO
}

\author{
Violence prevention and gender in Guanajuato state
}

Ricardo RODRÍGUEZ LUNA*

\begin{abstract}
Sumario:
Introducción I. Prevención y género: reorientaciones conceptuales II. Prevención de la violencia contra la mujer en el estado de Guanajuato III. Reflexión final. IV. Bibliografía
\end{abstract}

\begin{abstract}
Resumen: En este trabajo se analiza la definición de prevención de la violencia en los principales instrumentos jurídicos del estado de Guanajuato que declaran adoptar una perspectiva de género; se estudian sus elementos conceptuales y se indaga qué sentido adquiere al estar definida en normas y programas que emplean un enfoque de género.
\end{abstract}

Palabras clave: prevención, violencia, género, leyes, programas, Guanajuato, México.

Abstract. This paper analyzes the definition of prevention in gender law; are studied what it means to be defined by gender law in Guanajuato state.

Key words: prevention, violence, gender, law, Mexico, Guanajuato.

\section{Introducción}

La incursión de los estudios de género en la criminología adquirió notoriedad en el contexto de las transformaciones sociales de los años setenta del siglo pasado, situación que favoreció el desarrollo de nuevos enfoques y líneas de análisis en torno a la influencia de la diferencia sexual en la prevención, la comisión del delito y la seguridad pública.

En México, la idea de prevención adquirió nuevos e importantes matices particularmente en la década del año 200o, ya que se emitieron leyes, programas y/o planes de acción que declaran adoptar un enfoque de género; además, porque plantearon la prevención de la violencia ejercida habitualmente en contra de las mujeres por el hecho de serlo y no sólo la de un(os) delito(s) en particular. En este sentido fue significativa la emisión, el año 2007, de la Ley General de

\footnotetext{
Departamento de Derecho. Universidad de Guanajuato.
} 
Acceso de las Mujeres a una Vida Libre de Violencia; en el estado de Guanajuato, tres años después, se promulgó la Ley de Acceso de las Mujeres a una Vida Libre de Violencia de esta entidad.

La promulgación de leyes que adoptan una perspectiva de género ha supuesto diversos debates, algunos de estos vinculados específicamente al alcance y contenidos de la prevención, así como la determinación de estrategias para evitar el delito. Por tal motivo, en este trabajo cobra interés analizar el concepto de prevención de la violencia contra las mujeres contenida en diversos instrumentos del estado de Guanajuato que declaran adoptar un enfoque de género, como son la Ley de Acceso de las Mujeres a una Vida Libre de Violencia, el Reglamento de ésta y el Programa Integral para Prevenir, Atender, Sancionar y Erradicar la Violencia; la finalidad es estudiar los elementos conceptuales que la conforman e indagar qué sentido adquiere al estar definida por instrumentos legales que emplean una visión de género.

Para alcanzar el objetivo antes planteado, este artículo, en primer término, se esbozan algunas de las transformaciones más importantes que ha padecido el concepto de prevención del delito, particularmente desde los años setenta del siglo pasado. En segundo lugar, se hace una breve referencia a la forma en que se concibe la prevención en las principales leyes federales de México que declaran adoptar una perspectiva de género y cuyo objetivo es evitar la violencia contra la mujer; en tercero, se analizan este mismo tipo de leyes pero en el estado de Guanajuato.

\section{Prevención y género: reorientaciones conceptuales}

La convergencia de los estudios de género y criminológicos acontecida en el transcurso de la segunda mitad del siglo pasado, entre otras cuestiones, favoreció un cambio epistemológico en la investigación en torno al delito y su prevención, reorientó las prácticas, la legislación y los discursos que allanarían el camino para la puesta en marcha de las políticas en la materia de las últimas décadas ${ }^{1}$. En este contexto, la definición de prevención se transformó en diversos sentidos, en este apartado, se destacan algunos de los cambios más significativos en torno a dicha noción.

La prevención del delito es una de las principales funciones declaradas del sistema de justicia, particularmente, el derecho penal y la pena se han considerado útiles para evitar la comisión o la reincidencia de conductas ilegales. Desde esta perspectiva existen diversas teorías que explican el fundamento y fin de las penas ${ }^{2}$, mismas que conciben la prevención como una actuación estatal que tiene lugar una vez que el ilícito ha ocurrido. Esta idea imperó durante buena parte del siglo pasado.

En la década de los años setenta, en primer término, la prevención del delito se consolidó como una intervención anterior a la conducta delictiva y no posterior a ella, se consideró más bien una práctica pro-activa y no reactiva ante el delito ${ }^{3}$. En este primer momento, las criminólogas feministas mostraron que las teorías explicativas de delito no se habían preocupado por indagar si la delincuencia femenina respondía a motivaciones diversas de la masculina. Cuando

\footnotetext{
${ }^{1}$ SMART, Carol (1989), Feminism and the power of law, London, Routledge and Kegan Paul; Carlen, Pat (1992), "Criminal women and criminal justice, the limits to, and potential of, feminist and left realist perspectives", Issues in realist criminology, London, Sage, pp. 51-69; Facio, Alda (1993), "El derecho como producto del patriarcado", Programa Mujer, Justicia y Género, sobre patriarcas, jerarcas, patrones y otros varones, Costa Rica, ILANUD.

2 Éstas van de la retribución a la prevención y se clasifican en teorías absolutas y relativas de la pena. Bustos, Juan y Hormazábal, Hernán (1997), Lecciones de derecho penal (Volumen I), Madrid, Trotta, p. 44 y ss.

3 BARAtTA, Alessandro (1998), Entre la política criminal de seguridad y la política social en países con grandes conflictos sociales y políticos, ALTER 4-5, pp. 1-49; PAVARINI, Massimo (1998), Necesidades en materia de seguridad y cuestión criminal, ALTER 4-5, pp. 51-77.
} 
lo habían hecho, en general, habían atribuido a la naturaleza femenina las causas de la delincuencia de las mujeres ${ }^{4}$.

Asumida la prevención como una intervención antes de que aconteciera el delito, en segundo lugar, se desarrollaron estrategias que centraron su atención en el mejoramiento de las condiciones sociales, se concibió así la llamada prevención social55. Sin embargo, ésta decayó y ganó terreno la prevención situacional ${ }^{6}$, lo cual implicó un giro conceptual de importancia, ya que se dejó de centrar la atención en los aspectos sociales y en quién comete el delito, en su lugar, el interés se enfoca en las oportunidades y en los entornos en dónde ocurre el hecho delictivo7. El desarrollo de estas estrategias favoreció el arraigó, entre otras, de tres ideas que conviene subrayar:

Por un lado, se destacó la necesidad de la cooperación multiagencial o coordinación de todas aquellas instituciones implicadas en el control del delito ${ }^{8}$; por otro, se consideró que además de las autoridades, era fundamental la participación de la comunidad en estrategias preventivas ${ }^{9}$. Finalmente, en la década de los noventa, se consolidó la concepción de inseguridad subjetiva: una cuestión son los delitos que de hecho acontecen en un lugar determinado y otra muy diferente es la percepción que las personas tienen respecto de tales delitos - que objetivamente acontecen en su entorno ${ }^{10}$.

En el contexto de estos cambios, en el ámbito de los estudios de género, se señaló que la política criminal, especialmente las estrategias de seguridad pública y de prevención, no tenían en cuenta las experiencias de las mujeres ${ }^{11}$. De manera particular se mostró que la seguridad había sido considerada una cuestión objetiva y, en consecuencia, configurada por elementos que poseían también esta cualidad: el delito callejero, sobre todo aquel que se dirige a la propiedad y en el espacio público; se centra en las personas desconocidas; y, esencialmente, basada en los registros de los delitos denunciados a las autoridades del sistema penal. Esto evidenció que la legislación y las políticas públicas habían supuesto que las experiencias de un hombre medio

4 Smart, Carol, op.cit. nota 1; CARlen, Pat, op.cit, nota 1; FACIO, Alda, op.cit. nota 1.

5 Robert, Philippe (2003), El ciudadano, el delito y el Estado, Barcelona, Atelier; Van Swaaningen, René (2002), "Hacia un nuevo concepto de seguridad comunitaria", Revista Catalana de Seguridad Pública, 11, pp. 221-242.

6 Felson, Marcus y Clarke, Ronald (2010), "Cuestiones éticas en torno a la prevención situacional del delito", Tendencias en la prevención del delito y sus límites. Privacidad y dignidad humana frente al uso de nuevas tecnologías, Argentina, BdeF, pp. 173-206.

7 Tal como señalan algunos de los principales teóricos de estos planteamientos: su objetivo es impedir la perpetración del delito, en lugar de detectar y sancionar a los infractores. Se dirige, no a eliminar las tendencias criminales [...] sino, simplemente, a reducir las oportunidades para el delito. FELSON y ClaRKe, op. cit. nota 6, p. 174.

8 Lea, Matthews y Young (1993), "El Estado y el control del delito. Enfoques relativos a la actividad diversificada de sus agencias", Sistema Penal e intervenciones sociales. Algunas experiencias en Europa, Barcelona, HACER, pp. $17-62$.

9 Al respecto se han destacado al menos dos posiciones, una destaca las obligaciones y responsabilidades de la ciudadanía (FELSON y CLARKE, op. cit. nota 6, pp. 195-196), la otra, subraya la precaria relación entre derechos y responsabilidades de la ciudadanía cuando ésta interviene en estrategias de seguridad, ya que hay una tendencia a poner más el acento en las responsabilidades. CraWford, Adam (2002), "Les polítiques de seguretat i de prevenció de la delinqüència a Anglaterra i al País de Galles: noves estratègies i nous projectes”, Revista Catalana de Seguretat Pública 11, pp. 83-122.

10 O’Malley, P. (1992), "Risk, power and crime prevention”, Economy and Society 21,3, pp. 252-275. Cabe precisar que esta percepción constituye una preocupación vinculada a la inseguridad como problema social y no tanto (aunque pueden estar relacionadas) a las propias experiencias (acontecidas o posibles) de victimización ante el delito. Al respecto, ver también Robert, Philippe (2006), "Seguridad objetiva y seguridad subjetiva”, Revista Catalana de Seguridad Pública 16, pp. 91-102.

${ }^{11}$ Pith, Tamar (2009), “Són posibles les polítiques democràtiques per a la seguretat?", Revista Catalana de Seguretat Pública, maig, pp. 27-46; Stanko, Elizabeth (2009), "Es pot reduir la por de la delinqüència que tenen les dones?”, Revista Catalana de Seguretat Pública. maig, pp. 47-6o. 
también representaban las necesidades de las mujeres ${ }^{12}$. En este sentido, se ha destacado la persistencia de una noción prototípica del derecho a la seguridad: aquella que define su contenido en función de las necesidades de los hombres-propietarios, en el espacio público y casi exclusivamente en lo relativo a la seguridad física ${ }^{13}$. Además, al concebirse esta dimensión subjetiva de la seguridad, entre otras cuestiones, se evidenciaron grandes diferencias en las percepciones en torno la inseguridad entre mujeres y hombres ${ }^{14}$.

En las décadas de 1990 y 2000 , en tercer término, aunque dependiendo de cada contexto particular, aconteció una importante cuestión: se sostuvo que además de tener en cuenta el género en las explicaciones en torno a la delincuencia, era necesario el empleo de esta misma perspectiva en el desarrollo de medidas, estrategias y políticas públicas preventivas ${ }^{15}$. Aspecto que actualmente es avalado por organismos internacionales como la Organización de las Naciones Unidas $^{16}$ o la Unión Europea ${ }^{17}$. De esta forma, los desplazamientos conceptuales de la noción de prevención en las últimas décadas, especialmente desde los años 90 y hasta la actualidad, han sido matizados desde la óptica del género. Desde esta perspectiva, la noción misma de seguridad pública/ciudadana ha sido criticada y se ha señalado la necesidad de su reformulación ${ }^{18}$.

En cuarto lugar, la progresiva incorporación de la óptica de género en las investigaciones en torno a la prevención de la violencia, ha favorecido el desarrollo de nociones que actualmente están muy presentes en el debate internacional, como es el caso de las ideas de riesgo y miedo al delito ${ }^{19}$.

En cuanto al riesgo ${ }^{20} \mathrm{y} / \mathrm{o}$ factores de riesgo, éste se vincula al cálculo — de probabilidades estadísticas - de que acontezcan determinadas conductas, así como las posibilidades de evitarlas. Esto requiere determinar en qué sitios y bajo qué condiciones es más probable que sucedan las

\footnotetext{
${ }^{12}$ Ídem.

${ }^{13}$ Bodelón, Encarna (2009), "Las mujeres y las nuevas legislaciones sobre sus derechos: el caso del derecho a la seguridad”, Revista Catalana de Seguridad Pública, mayo, pp. 79-91; Ver también, Naredo, María (2009), "Adecuación de las políticas públicas de seguridad a las necesidades de las mujeres: una cuestión urgente", Revista Catalana de Seguridad Pública, mayo, pp. 67-77;

${ }^{14}$ Piтh, Tamar, op. cit. nota 11.

${ }^{15}$ Ídem.

${ }^{16}$ Organización de las Naciones Unidas (2013), Informe sobre el $57^{\circ}$ período de sesiones (doc. E/2013/27E/ CN.6/2013/11). Nueva York, ONU (2010), Informe de la reunión del grupo intergubernamental de expertos encargados de examinar y actualizar las Estrategias y Medidas Prácticas Modelo para la Eliminación de la Violencia contra la Mujer en el campo de la prevención del Delito y la Justicia Penal (doc. E/CN.15/2010/2). Nueva York, ONU.

${ }^{17}$ Unión Europea (2012), Directiva por la que se establecen normas mínimas sobre los derechos, el apoyo y la protección de las víctimas de delitos (directiva 2012/29/UE, Bruselas, UE; (2010), Programa de Estocolmo 2010-2014. Una Europa abierta y segura que sirva y proteja al ciudadano (doc. 2010/C 115/o1). Bruselas, UE (2002), Recomendación [Rec (2002)5], Comité de Ministros del Consejo de Europa. Bruselas, UE.

${ }^{18}$ Consejo de Municipios y Regiones de Europa (2005), Carta Europea para la Igualdad de Mujeres y Hombres en la Vida Local.

${ }^{19}$ O’MALLEY, op. cit. nota 10.

${ }^{20}$ Los planteamientos en torno al riesgo en el ámbito criminológico, si bien adoptan diversos principios o nociones de la sociedad del riesgo, poseen un marcado carácter técnico-científico que relaciona la delincuencia con cuestiones como los cálculos econométricos, variables estadísticas, indicadores, bases de datos, probabilidad, predicción, peligro y/o precaución ante el delito. Van Swaaningen, René, (2005), "La política de seguridad ciudadana en Holanda: Traficando con el miedo", Revista Española de Investigación Criminológica AC-02-05, pp. 1-21; ver también, Da Agra, Candido, et.al. (2003, eds), La seguridad en la sociedad del riesgo: un debate abierto, Barcelona, Atelier; Mendoza, B., (2003), "Gestión del riesgo y política criminal de seguridad en la sociedad del riesgo", La seguridad en la sociedad del riesgo. Un debate abierto, Barcelona, Atelier, pp. 67-89; Recasens, Amadeu, (2003), "Globalización, riesgo y seguridad: el continuóse de que alguien empezóse", La seguridad en la sociedad del riesgo. Un debate abierto, Barcelona, Atelier, pp. 365-379.
} 
conductas delictivas ${ }^{21}$. De igual forma, los perfiles de riesgo, pretenden determinar a quiénes debería dirigirse un programa determinado y en dónde cabe desarrollarlo. En este sentido, consiste en evaluar las probabilidades que tienen determinados colectivos de padecer algún delito ${ }^{22}$. De esta forma, puede decirse que a través de cálculos se especifican riesgos determinados, es decir, eventos no ocurridos pero que es probable acontezcan ${ }^{23}$.

En el ámbito de los estudios de género, de manera particular E. Stanko, ha criticado el concepto de riesgo al plantear una cuestión básica: ¿qué hacemos aflorar cómo riesgo? ${ }^{24}$, especialmente si se reconoce el miedo al delito de las mujeres, si no se tiene en cuenta el contexto de la incertidumbre de género, si no se sabe cómo se definen los indicadores que determinan la llamadas poblaciones afectadas o los criterios de elaboración de lo que constituye un peligro, o bien, cuando el debate se centra particularmente en aquellos riesgos que se pueden evitar individualmente, es decir, cuando la respuesta a los riesgos se enfoca como una cuestión de prudencia individual y no colectiva.

El miedo al delito, por su parte, a diferencia de la inseguridad subjetiva - que se relaciona a la percepción de la inseguridad, como problema social en general一, se vincula de manera específica a la creencia/sentimiento que tiene una persona acerca de la posibilidad de ser víctima de un delito. De acuerdo con Hale ${ }^{25}$, esto se vincula a diversos elementos como el género, la edad, la clase social, la pertenencia a una etnia determinada. No obstante, también se ha precisado la complejidad y dificultades en la medición o determinación de dichos miedos ${ }^{26}$.

Las investigaciones en el ámbito de la violencia de género señalan que la diferencia de género es la conclusión más presente en las publicaciones sobre el miedo a la delincuencia ${ }^{27}$. Se ha destacado que las mujeres adoptan de manera consciente e inconsciente rutinas y/o hábitos para evitar determinados riesgos, cambios que se vinculan a los procesos de socialización y la forma en que interiorizan el miedo y la evitación de dichos riesgos, muchas veces en los espacios públicos. Esta situación tiene una consecuencia de importancia, ya que los cambios de costumbres o hábitos implican prohibiciones y censuras que limitan la libertad femenina más que la masculina: pero, si lo miramos desde otro punto de vista, configuran un clima generalizado de «victimización»o, mejor, quizás, indican una situación que se puede definir tranquilamente como «opresiva» ${ }^{28}$.

Un aspecto que ha recibo críticas importantes ha sido el de las formas e instrumentos de medición del miedo al delito ${ }^{29}$. En el ámbito de los estudios de género se ha destacado que éstos interpretan los temores de las mujeres con base en peligros preconcebidos y enraizados en peligros concretos, esencialmente representados a través de hombres desconocidos y en el espacio

\footnotetext{
${ }^{21}$ DA Agra, Candido, op.cit. nota 20.

22 VAN SWAANIngen, René (2011), Perspectivas europeas para una criminología crítica, Argentina, BdeF: p. 284.

23 EwAld, F. (1991), "Insurance and risk", The Foucault effect: studies in governmentality, London, Harvester Weatsheaf, pp. 197-210.

${ }^{24}$ STANKo, Elizabeth, op. cit. nota 11, pp. 61-62.

${ }^{25}$ Hale, C. (1996), "Fear of Crime. A Review of the Literature", International Review of Victimology, 4, 2, pp. 79150.

${ }^{26}$ SPARKs, R. (2007), "Perspectivas sobre el riesgo y política penal”, Delito y Sociedad 26(23), pp. 29-47; Harcourt, B. E. (2007), Against prediction: profiling, policing, and punishing in an actuarial age. Chicago, University of Chicago Press; Haghighi, B. and Sorensen, J., (1996), “America's fear of crime”, Americans View Crime and Justice: A National Public Opinion Survey, USA, Sage Publications, pp. 16-30; WARr, M., (1984), "Fear of victimization: Why are women and the elderly more afraid?", Social Science Quarterly 65, pp. 681-702.

27 Stanko, E, op. cit. nota 11, p. 53.

${ }^{28}$ Pitch, T, op. cit. nota 11, p. 35.

${ }^{29}$ SPARKS, op. cit. nota 26; Harcourt, op. cit. 26.
} 
público. Por tanto, más allá del tradicional ámbito público/privado, deben considerarse también las relaciones que producen inseguridad y violencia; es necesario tener en cuenta las fuentes de inseguridad en la experiencia de las mujeres, especialmente las que se vinculan a comportamientos de personas conocidas, así como la socialización femenina de la precaución-miedo ${ }^{30}$. De igual manera, se ha señalado la necesidad de generar un nuevo modelo de derechos y de justicia, dado que, si nos interesamos en serio por las dificultades de la mitad de la población urbana, descubrimos que esta mitad reclama y tiene necesidad de más libertad, y no de más protección ${ }^{31}$.

\section{Prevención de la violencia contra la mujer en el estado de Guanajuato}

La preocupación por una mayor y más eficaz prevención de la violencia ha cobrado una gran relevancia en las últimas décadas en México, así como su inclusión en diversas leyes, planes y programas de gobierno ${ }^{32}$ que inciden en esta materia, algunos de los cuales declaran adoptar una perspectiva de género. En este apartado, en primer término, se esboza la evolución del concepto en el marco de la federal mexicano; en segundo, de manera particular, se analizan los instrumentos más relevantes en materia de prevención de la violencia contra las mujeres en el estado de Guanajuato.

\subsection{Definición de prevención en México}

En el transcurso de la segunda mitad del siglo pasado en México, conforme a los cambios señalados en el apartado previo, puede decirse que la prevención se incorporó a la política criminal mexicana, bajo diversas características:

Estuvo ligada indisolublemente, en el período de los años setenta a noventa, a la función policial, así como a la constante exigencia de la participación ciudadana ${ }^{33}$; las referencias a conceptos como inseguridad subjetiva, riesgo o género fueron escasos sino es que prácticamente ausentes. En términos generales, las leyes, instrumentos y programas en torno a la seguridad se destacaron por una ideología de castigo, reactiva ante el delito ${ }^{34}$.

En el transcurso de la década del año 2000, aunque la idea de prevención continúa ligada de forma importante a la función policial y a la participación de la ciudadanía ${ }^{35}$, se incorporaron de

${ }^{30}$ NAREdo, María, op. cit. nota 15, pp. 72-73

${ }^{31}$ Piтch, T. op. cit. nota 11, p. 43; ver también Bodelón, E. op. cit. nota 13.

${ }^{32}$ Por ejemplo, entre otros: la Constitución Política de los Estados Unidos Mexicanos y la Ley de seguridad pública del DF. Además, se han emitido: el Programa Nacional de Seguridad Pública (PNSP) 1995-2000; el año de 1995, por primera vez, se emitió un Programa de Seguridad Pública del DF (PSPDF) 1995-200o. Actualmente, se han sido emitidas diversas ediciones del Programa Nacional de Seguridad Pública (PNSP): 2001-2006; 2008-2012 y 2014-2018; y, también del Programa de Seguridad Pública del DF (PSPDF): 2007-2012 y El Programa Sectorial de Seguridad Ciudadana del Distrito Federal, 2014-2018.

33 El PSPDF 1995-2000, por ejemplo, concibe la seguridad como un reto que precisa de la participación de la ciudadanía, y para ello, se propone el establecimiento de los mecanismos que permitan hacer efectiva tal participación. Departamento del Distrito Federal, (1995), "Programa de Seguridad Pública para el Distrito Federal (PSPDF) 1995-2000”, Diario Oficial de la Federación DVI, 19, primera sección, pp. 25-27.

34 Pérez, Agustín (2002), Análisis y evaluación de leyes en materia de prevención delictiva, México, FCE/UAM, p. 12; el subrayado es mío.

35 Por ejemplo, el Programa General de Desarrollo del DF 200o-20o6 señala: Los buenos resultados en materia de seguridad pública y prevención del delito se obtienen mediante la estrecha colaboración de los ciudadanos con la policía para llevar a cabo operativos de prevención y respuesta inmediata, ya que a través de éstos se reducen sensiblemente los factores de riesgo de victimización, así como los índices delictivos. Gobierno del Distrito Federal, 
lleno las ideas de inseguridad subjetiva ${ }^{36}$, delitos de alto impacto social ${ }^{37}$, la noción de prevención situacional ${ }^{38}$ y se prestó mayor atención a las víctimas del delito ${ }^{39}$. Además, cabe destacar dos cambios sustanciales:

Por un lado, se consolidó la noción de riesgo y la reducción de éstos se convirtió en parte fundamental de la estrategia preventiva ${ }^{40}$; por otro, la noción de género irrumpió en el escenario jurídico y político en torno a la seguridad y la prevención ${ }^{41}$. En este sentido, representa un punto de inflexión la Ley General de Acceso de las Mujeres a una Vida Libre de Violencia (LGAMVLV), promulgada el año 2007. En su exposición de motivos se reconoce la necesidad de contar con un instrumento jurídico que contenga una perspectiva de género.

En cuanto a la definición de prevención de la violencia contra la mujer, ésta aparece delimitada esencialmente por la LGAMVLV y su Reglamento, a su vez, el Programa Integral para Prevenir, Atender, Sancionar y Erradicar la Violencia contra las Mujeres 2014-2018 (Programa Integral), constituye la principal estrategia operativa que pone en práctica un buen número de acciones preventivas. Estos instrumentos, de hecho, conforman el marco guía de la actuación preventiva más importantes en torno a la prevención en el ámbito de la violencia de género en México.

Una cuestión muy importante es que dichos instrumentos declaran adoptar una perspectiva de género, sin embargo, ni la LGAMVLV ni el Programa Integral 2014-2018 ${ }^{42}$ definen qué es la

(2001), "Programa General de Desarrollo del Distrito Federal (PGDDF) 2001-2006", Gaceta Oficial del Distrito Federal, 141, p. 42. En el mismo sentido, ver también el PNSP 2001-2006.

${ }^{36}$ El PSPDF 2007-2012, en su diagnóstico de la inseguridad, sostiene: El resultado de lo anterior, se ha traducido en un abatimiento de los indicadores de la actividad delictiva, que no necesariamente de la percepción social, de la seguridad - inseguridad pública, porque los delitos de alto impacto, asociados a la violencia y por lo general los delitos del orden federal, son los que dejan la huella del temor y sus secuelas en la población. Gobierno del Distrito Federal, (2008), "Programa de Seguridad Pública para el Distrito Federal (PSPDF) 2007-2012. Seguridad y justicia para la Ciudad de México", Gaceta Oficial del Distrito Federal 250, p. 133.

${ }^{37}$ Este tipo de delitos está asociado con el homicidio doloso, secuestro, extorsión, trata de personas y robo con violencia, homicidios de odio y feminicidio. Secretaría de Gobernación, (2014), "Programa Nacional de Seguridad Pública (PNSP) 2014-2018”, Diario Oficial de la Federación DCCXXVII, 25, cuarta sección, pp. 66-70.

${ }^{38}$ La prevención en el ámbito situacional consiste en modificar el entorno para propiciar la convivencia y la cohesión social, así como disminuir los factores de riesgo que facilitan fenómenos de violencia y de incidencia delictiva, mediante [...] Ibídem, p. 95.

39 Por ejemplo, el PNSP plantea como su estrategia no. 1.4: Fortalecer y consolidar la red nacional de atención a víctimas del delito. Secretaría de Seguridad Pública (2009), "Programa Nacional de Seguridad Pública (PNSP) 2008-2012", Diario Oficial de la Federación DCLXVI, 18, primera sección, pp. 24-25.

$4^{40}$ Ver, por ejemplo, en el programa de gobierno del antiguo Distrito Federal, en donde aparecía la idea de riesgo vinculada a colectivos específicos: pandillas, jóvenes y adolescentes. Gobierno del Distrito Federal, op. cit. nota 35. pp. 31-57. Por otro lado, la estrategia 4.2 del actual PNSP consiste en Fomentar el uso de "Inteligencia Social", como complemento de la inteligencia policial, para identificar factores de riesgo para la seguridad. Secretaría de Gobernación, op. cit, nota 37, p. 73.

${ }^{41}$ Por ejemplo, la estrategia 4.7 del actual PNSP consiste en Incorporar la "Perspectiva de Género" en la actuación de las instituciones de seguridad pública, de manera transversal. Entre sus líneas de acción destacan, entre otras: Promover el intercambio de información y experiencias internacionales sobre incorporación de la "Perspectiva de Género" en políticas de seguridad pública, o bien, Impulsar la incorporación de la "Perspectiva de Género" de manera transversal en la normatividad sobre seguridad y justicia. Ibídem, p. 76.

${ }^{42}$ En la primera edición de dicho programa, publicado por la Secretaría de Gobernación, sí se plantearon algunas cuestiones conceptuales, aunque muy generales, en torno a la prevención: la prevención es fundamental y posee un carácter integral que vincula todas las acciones del Estado. Así, la prevención es jurídica, política, criminológica, administrativa o sociocultural y tiene distinto alcances y niveles, pero su fin último es garantizar los derechos humanos de las mujeres y evitar su afectación mediante la comisión de conductas delictivas. Secretaría de Gobernación, (s/f), Programa Integral para Prevenir, Atender, Sancionar y Erradicar la Violencia contra las Mujeres, México, SG., p. 10 (el subrayado es mío). Así mismo, se sostiene que la prevención consiste en transfor- 
prevención y tampoco indican si adquiere un significado particular prevenir desde dicha óptica. El Reglamento de la ley es en donde se explica en qué consiste la prevención de la violencia contra la mujer ${ }^{43}$, se prevé el desarrollo de un Modelo de prevención ${ }^{44} \mathrm{y}$, conceptualmente, la noción de riesgo pasa a ser una parte fundamental de la noción de prevención. No obstante, no se define qué es riesgo o factores de riesgo, y aunque señala qué es un estado de riesgo, se hace de forma muy general: cualquier circunstancia que haga previsible una situación de Violencia contra las Mujeres ${ }^{45}$. El Programa Integral (2014-2018), por su parte, tampoco aporta un concepto de riesgo ni de prevención, aunque evidentemente, el uso que hace de esta última noción está vinculada a aquella plasmada en el Reglamento de la LGAMVLV.

\subsection{Definición de prevención en el estado de Guanajuato}

En el Estado de Guanajuato existen diversas leyes y programas que regulan variados aspectos en torno a la prevención de la violencia ${ }^{46}$, sin embargo, la Ley de Acceso de las Mujeres a una Vida Libre de Violencia (LAMVLVGTO) ${ }^{47}$ y su Reglamento ${ }^{48}$, así como el Programa Estatal para Prevenir, Atender, Sancionar y Erradicar la Violencia contra las Mujeres ${ }^{49}$, constituyen una parte conceptual fundamental de tal definición; además, expresamente se establece que el enfoque de género orientará las políticas públicas en materia de acceso a una vida libre de violencia de las

mar los patrones socioculturales de comportamiento de mujeres y hombres, lo cual se pretende alcanzar a través de la generación de políticas públicas de prevención que disminuyan los factores de riesgo de la violencia, a fin de garantizar a las mujeres sus derechos y libertades. Ídem, p. 34.

43 Conjunto de estrategias para que los tres órdenes de gobierno con la participación social, generen condiciones idóneas a efecto de erradicar la violencia y cualquier forma de discriminación hacia la mujer, en los ámbitos públicos y privados, y modifiquen los patrones de comportamiento basados en estereotipos de hombres y mujeres. Secretaría de Gobernación, (2008), "Reglamento de la Ley General de Acceso de las Mujeres a una Vida Libre de Violencia”, Diario Oficial de la Federación DCLIV, 8, primera sección, art., 4 fracc. I.

44 El conjunto de acciones encaminadas a promover y difundir los derechos de las mujeres e identificar factores de riesgo con el fin de evitar actos de violencia. [Este modelo se integra por diversas acciones]: I. Sensibilizar concientizar y educar para prevenir la violencia; II. Diseñar campañas de difusión para reducir el número de víctimas; III. Detectar en forma oportuna los posibles actos o eventos de Violencia; VI. Todas aquellas medidas y acciones que sean necesarias para eliminar los factores de riesgo de Violencia contra las Mujeres. Ibídem, artículo 10 (las negritas son mías).

45 Aspecto señalado en el (artículo, 2 fracción VI). Además, el reglamento señala diversos aspectos a tener en cuenta para la ejecución del modelo de prevención: los niveles primario, secundario y terciario en las intervenciones; la percepción social de la violencia hacia las mujeres; los usos y costumbres de los pueblos indígenas; el grado de pobreza, marginación y analfabetismo, así como la esperanza de vida de la población a la que va dirigida; la intervención interdisciplinaria; y, la información desagregada, entre otros, por sexo, edad, lugar de los hechos de violencia, antecedentes de violencia, tipos de delitos, nivel educativo, condición socioeconómica, grupos en situación de vulnerabilidad y origen étnico. Ibídem, art. 11.

${ }^{46}$ Entre otros, cabe destacar: a). Ley para la Igualdad entre Mujeres y Hombres; b). Ley para Prevenir, Atender y Erradicar la Violencia; c). Ley del Sistema de Seguridad Pública; y, d). Ley de Acceso de las Mujeres a una Vida Libre de Violencia para el Estado de Guanajuato (LAMVLVGTO) y su Reglamento; e). Programa Estatal para Prevenir, atender y Erradicar la Violencia en el Estado de Guanajuato; f). Programa Estatal para Prevenir, Atender, Sancionar y Erradicar la Violencia contra las Mujeres; y, g). Programa Estatal de Prevención Social. Actuar es Prevenir.

47 Gobierno del Estado de Guanajuato (2010), "Ley de Acceso de las Mujeres a una Vida Libre de Violencia para el Estado de Guanajuato”, Periódico Oficial, Gobierno del Estado, Guanajuato, Gto. no. 189.

${ }^{48}$ Gobierno del Estado de Guanajuato (2011), "Reglamento de la Ley de Acceso de las Mujeres a una Vida Libre de Violencia para el Estado de Guanajuato”, Periódico Oficial, Gobierno del Estado, Guanajuato, Gto. no. 142

49 Gobierno del Estado de Guanajuato (2017), Programa Estatal para Prevenir, Atender, Sancionar y Erradicar la Violencia contra las Mujeres, México, IMUG. 
mujeres ${ }^{50}$, para ello, se regirán por diversos principios rectores, como la igualdad jurídica entre la mujer y el hombre, y la no discriminación ${ }^{51}$.

En el Reglamento de la LAMVLVGTO se definen los ejes estratégicos de la implementación de políticas públicas en materia de violencia contra las mujeres: prevención, atención, sanción y erradicación ${ }^{52}$. El primero de estos ejes persigue el objetivo de reducir proactivamente los factores de riesgo de violencia contra las mujeres ${ }^{53}$, para alcanzar esta meta se proponen dos elementos: por un lado, anticipar y evitar la generación de violencia contra las mujeres en todos los tipos y ámbitos previstos por la Ley de Acceso $0^{54}$. Por otro, capacitar y sensibilizar a las mujeres para que adviertan sus situaciones de riesgo, así como acercar a ellas los medios o instituciones para hacer efectivos sus derechos ${ }^{55}$.

Cabe destacar que el Reglamento define la prevención en términos de factores de riesgo, sin embargo, no establece qué es un riesgo ni especifica cuáles son éstos, en qué consisten o cómo se determinan. Además, cuestión muy importante, para alcanzar el objetivo de la prevención, las políticas públicas se orientarán a capacitar y sensibilizar a las mujeres para que adviertan sus situaciones de riesgo. Es decir, son las propias mujeres quienes advertirán los riesgos, en última instancia, son ellas quienes deben responsabilizarse de identificar los riesgos y evitar así la violencia. La noción de prevención acaba por depositar en las mujeres una parte sustancial de la misma ley, es decir, evitar la violencia.

Se sostiene también que la prevención deberá tener en cuenta diversos aspectos, entre otros, el tipo y ámbito de violencia a prevenir y la población a la que está dirigida ${ }^{56}$, así como también los mecanismos de evaluación para generar los indicadores ${ }^{57}$. No obstante, aunque las mujeres son el sector de la población a quienes se deben dirigir las acciones preventivas, no se especifican elementos diferenciadores como la edad, si viven en ámbito rural o urbano, si son indígenas, etc.

Por otra parte, la LAMVLVGTO prevé el Programa Estatal para Prevenir, Atender, Sancionar y Erradicar la Violencia contra las Mujeres ${ }^{58}$. Éste, con base en su diagnóstico estratégico ${ }^{59}$, sostiene que el problema a que se enfrenta es la insuficiencia de acciones de prevención, atención y sanción encaminadas a erradicar la violencia contra las mujeres ${ }^{60}$. De esta forma, se establecen las líneas estratégicas de la política pública que permitirán atender la violencia contra las mu-

${ }^{50}$ Gobierno del Estado de Guanajuato, op. cit. nota 47, artículo 1. Así mismo, cabe señalar que

la perspectiva de género se define como la visión científica, analítica y política sobre las mujeres y los hombres, que propone eliminar las causas de opresión de género como la desigualdad, la injusticia y la jerarquización de las personas basada en el género. Promueve la igualdad, la equidad, el adelanto y el bienestar de las mujeres; contribuye a construir una sociedad en donde las mujeres y los hombres tengan el mismo valor, la igualdad de derechos y oportunidades, para acceder al desarrollo social y la representación en los ámbitos de toma de decisiones. Ibídem, artículo 2.

${ }^{51}$ Ibídem, artículo 3 fracción I y III.

52 Gobierno del Estado de Guanajuato, op. cit. nota 48, artículo 10.

${ }^{53}$ Ibídem, artículo 11.

54 Ibídem, artículo 11 fracc. I.

55 Ibídem, artículo 11 fracc II.

${ }^{56}$ Ibídem, artículo 12 fracc. I.

57 Ibídem, artículo 12 fracc. IV.

${ }^{58}$ Este es definido como el mecanismo que contiene los objetivos, acciones, metas, estrategias y responsables que de forma planeada y coordinada deberán realizar las dependencias y entidades de la administración pública, en corto, mediano y largo plazo, para la prevención, atención sanción y erradicación de la violencia contra las mujeres. Gobierno del Estado de Guanajuato, op. cit. nota 48, artículo 12.

59 Gobierno del Estado de Guanajuato, op. cit, nota 49, p. 26.

${ }^{60}$ Ídem 
jeres $^{61}$. En el ámbito de la prevención se plantean un conjunto de acciones a desarrollar, metas a alcanzar, así mismo, se indican las entidades responsables de lograrlo. Sin embargo, llama la atención que no se establecen cuáles son los riesgos tenidos en cuenta para plantear tales acciones, de hecho, prácticamente no se hace referencia a la noción de factores de riesgo.

Antes de concluir este apartado, cabe hacer una breve referencia a la Ley del Sistema de Seguridad Pública ${ }^{62}$, ya que expresamente sostiene que el género es uno de los principios a tener en cuenta en materia de seguridad y prevención. Al respecto, se señala que las políticas públicas deberán centrar su atención, entre otros, en las niñas y los niños, las mujeres, así como las y los jóvenes en situación de riesgo ${ }^{63}$; $y$ deberán de considerar las necesidades y circunstancias específicas determinadas, por ejemplo, el género [...] y las necesidades de grupos vulnerables o en riesgo ${ }^{64}$. Además, dado que la ley persigue, entre otras, la finalidad de disminuir y contener la incidencia delictiva, identificando sus factores criminógenos ${ }^{65}$, es destacable que no define qué es la prevención, factores criminógenos ni tampoco qué consideraciones se tuvieron en cuenta para determinar los grupos en riesgo y sus necesidades.

Una situación similar acontece en diversos instrumentos legales, como Ley para Prevenir, Atender y Erradicar la Violencia en el estado de Guanajuato ${ }^{66}$ o el Programa Estatal de Prevención Social. Actuar es Prevenir, mismo que constituye el instrumento a través del cual se despliegan las políticas públicas en el ámbito de prevención social de la violencia y la delincuencia, tienen como principal propósito la identificación y tratamiento de aquellos factores de riesgo que originan conductas contrarias al orden y la paz social ${ }^{67}$. La noción de prevención social aparece sustentada en la noción de factores de riesgo (y de participación ciudadana), sin embargo, no se define qué o cuáles son éstos, aunque se plantea un cuestión de interés: la prevención social actúa sobre aquellos factores de riesgo de carácter estructural como lo son la pobreza, marginación, deserción escolar, inseguridad, desempleo, insalubridad, etc. ${ }^{68}$ No obstante, no se establece si

\footnotetext{
${ }^{61}$ Ibídem, pp. 55-69

${ }^{62}$ Gobierno del Estado de Guanajuato, (2014), “Decreto no. 191. Ley del Sistema de Seguridad Pública del Estado de Guanajuato", Periódico Oficial, Gobierno del Estado, Guanajuato, Gto. no. 188.

${ }^{6}$ Ibídem, artículo 4, fracción III.

${ }^{64}$ Ibídem, artículo 4, fracción VII.

${ }^{65}$ Ibídem, artículo 3, fracción II.

${ }^{66}$ Esta ley no entra a definir los aspectos que aquí interesan y remite a otras ordenamiento y/o instancias; en lo que respecta al género, aunque no establece expresamente la inclusión de esta perspectiva, se rige por principios como el respeto a la vida, a la dignidad humana y a una vida libre de violencia, la igualdad y la equidad (artículo 5). Por otra parte, la prevención es definida como el conjunto de acciones, medidas y estrategias encaminadas a impedir que se genere o continúe generando violencia (artículo 49); y sostiene que el Consejo Estatal aprobará modelos de prevención, atención y erradicación de la violencia para la implementación de las políticas públicas en la materia (artículo 11). Gobierno del Estado de Guanajuato, (2009), "Ley para prevenir, atender y erradicar la violencia en el Estado de Guanajuato”, Periódico Oficial, Gobierno del Estado, Guanajuato, Gto. no. 50.

67 Secretaría de Seguridad Pública del Estado de Guanajuato (s.f.), Programa Estatal de Prevención Social. ACTUAR ES PREVENIR, México, p. 3 (el subrayado es mío). Además, el Programa Estatal destaca el aspecto de los factores de riesgo, en este sentido, cuando se explica la importancia del programa, se sostiene:
}

\footnotetext{
Es por ello que la prevención social de la violencia y la delincuencia es uno de los componentes fundamentales en los que descansa el bienestar social del Estado. Con tal propósito, se ha conformado el presente instrumento de planeación, atendiendo a la capacidad institucional y operativa de las dependencias encargadas de brindar seguridad pública, con lo cual se podrán llevar a cabo políticas encaminadas a reducir los factores de riesgo que posibiliten la generación de la violencia y delincuencia, así como mitigar las causas que las generan, Ibídem, p. 5 (el subrayado es mío).
}

${ }^{68}$ El mismo Programa sostiene que la prevención social actúa sobre aquellos factores de riesgo de carácter estructural como lo son la pobreza, marginación, deserción escolar, inseguridad, desempleo, insalubridad, etc.; todo lo anterior, mediante políticas públicas con plazos razonables, siempre tendiendo a la satisfacción de aquellos reque- 
dichos riesgos actúan diferenciadamente, ni cómo, en hombres y mujeres; tampoco se específica si hay riesgos de carácter no estructural sobre los que actúen los mismos u otros instrumentos legales.

\section{Reflexión final}

La Ley de Acceso de las Mujeres a una Vida Libre de Violencia del Estado de Guanajuato, su Reglamento y el Programa Estatal para Prevenir, Atender, Sancionar y Erradicar la Violencia contra las Mujeres, constituyen el eje fundamental del concepto de prevención de la violencia contra la mujer definido desde una óptica de género. El riesgo y/o factores de riesgo y su minimización constituyen las ideas clave de la prevención, así como de las estrategias y acciones contenidas en dichos instrumentos.

No obstante, el riesgo y/o factor de riesgo no se definen de forma clara ni precisa, por un lado, de manera tautológica, se propone anticipar y evitar generación de violencia para alcanzar la prevención; por otro, en última instancia se responsabiliza a las mujeres de advertir sus situaciones de riesgo. De esta forma, el concepto de riesgo se concibe como una cuestión de cautela o moderación individual.

Tampoco se establece claramente cómo se evalúan los riesgos para las mujeres, ni los indicadores que verifiquen el cumplimiento de los objetivos en términos de mayor seguridad. De esta forma, resulta problemático e impreciso el establecimiento de aquello que constituye un riesgo, su cálculo, su influencia en las acciones preventivas concretas, así como su impacto en la seguridad de las mujeres. Todo ello se hace suponiendo que no hay afectación a las libertades fundamentales. Además, el concepto de prevención no adquiere un sentido particular ni matices relevantes al contenerse en leyes y programas que adoptan una visión de género.

Las leyes y programas analizados emplean la noción de género como un sinónimo de mujer o de acciones para éstas, ya que, de facto, los instrumentos analizados no consideran necesaria una estrategia orientada a los varones. Se podría sostener que los objetivos de las políticas de violencia contra las mujeres, dado su mismo origen y evolución, no se orientan a los hombres y que por ello no habría que implementar acciones específicas. No obstante, cabe cuestionar la suficiencia de instrumentos jurídicos y políticos cuyo objetivo es la prevención de la violencia contra las mujeres, pero que a su vez, prácticamente no dirigen medidas orientadas a los principales responsables de la violencia que se pretende prevenir, combatir y erradicar. Además, la misma estrategia preventiva de violencia contra las mujeres, entre otras cuestiones, plantea la importancia de modificar estereotipos, de considerar diferenciadamente riesgos y modificar patrones de comportamiento de hombres y mujeres.

\section{Bibliografía}

Baratta, Alessandro (1998), "Entre la política criminal de seguridad y la política social en países con grandes conflictos sociales y políticos", ALTER 4-5, pp. 1-49.

Bodelón, Encarna (2009), "Las mujeres y las nuevas legislaciones sobre sus derechos: el caso del derecho a la seguridad", Revista Catalana de Seguridad Pública, mayo, pp. 79-91.

rimientos mínimos de bienestar social y con el apoyo de los diferentes grupos sociales que conforman el sistema social. Ibídem, p. 59. 
Bustos, Juan y Hormazábal, Hernán (1997), Lecciones de derecho penal (Volumen I), Madrid, Trotta.

CARlen, Pat (1992), "Criminal women and criminal justice, the limits to, and potential of, feminist and left realist perspectives", Issues in realist criminology, London, Sage, pp. 51-69.

Consejo de Municipios y Regiones de Europa (2005), Carta Europea para la Igualdad de Mujeres y Hombres en la Vida Local. Bruselas, UE.

Crawford, Adam (2002), "Les polítiques de seguretat i de prevenció de la delinqüència a Anglaterra i al País de Gal.les: noves estratègies i nous projectes", Revista Catalana de Seguretat Pública 11, pp. 83-122.

(1998). Crime prevention and community safety: politics, policies and practices. Londres, Longman.

Da Agra, C. et.al. (2003, eds), La seguridad en la sociedad del riesgo: un debate abierto. Barcelona, Atelier.

EwALD, F. (1991), "Insurance and risk", The Foucault effect: studies in governmentality, London, Harvester Weatsheaf, pp. 197-210.

FACIO, Alda (1993), "El derecho como producto del patriarcado", Programa Mujer, Justicia y Género, sobre patriarcas, jerarcas, patrones y otros varones, Costa Rica, ILANUD.

Felson, M. y Clarke, R. (2010), "Cuestiones éticas en torno a la prevención situacional del delito". En Tendencias en la prevención del delito y sus límites. Privacidad y dignidad humana frente al uso de nuevas tecnologías, Argentina, BdeF, pp. 173-206.

Haghighi, B. And Sorensen, J. (1996), “America’s fear of crime”, Americans View Crime and Justice: A National Public Opinion Survey, USA, Sage Publications, pp. 16-30.

Harcourt, B. E. (2007), Against prediction: profiling, policing, and punishing in an actuarial age, Chicago, University of Chicago Press.

LeA, Matthews y Young (1993), "El Estado y el control del delito. Enfoques relativos a la actividad diversificada de sus agencias", Sistema Penal e intervenciones sociales. Algunas experiencias en Europa, Barcelona, HACER, pp. 17-62.

Mendoza, B. (2003), "Gestión del riesgo y política criminal de seguridad en la sociedad del riesgo", La seguridad en la sociedad del riesgo. Un debate abierto, Barcelona, Atelier, pp. 67-89.

NAredo, María (2011), "Metodologías para la detección de problemas de seguridad con enfoque de género", No surtis sola. Espais publics segurs amb perspectiva de gènere, Barcelona, ICPS, pp. 11-48.

(2009), “Adecuación de las políticas públicas de seguridad a las necesidades de las mujeres: una cuestión urgente", Revista Catalana de Seguridad Pública mayo, pp. 67-77. 
O’Malley, P. (1992), “Risk, power and crime prevention”, Economy and Society 21,3, 252-275.

Organización de las Naciones Unidas, (2013), Informe sobre el $57^{\circ}$ período de sesiones (doc. E/2013/27E/CN.6/2013/11), Nueva York, ONU.

(2010), Informe de la reunión del grupo intergubernamental de expertos encargados de examinar y actualizar las Estrategias y Medidas Prácticas Modelo para la Eliminación de la Violencia contra la Mujer en el campo de la prevención del Delito y la Justicia Penal (doc. E/CN.15/2010/2), Nueva York, ONU.

Pavarini, Massimo (2006), Un arte abyecto. Ensayo sobre el gobierno de la penalidad. Argentina, AD·HOC.

(1998), "Necesidades en materia de seguridad y cuestión criminal", ALTER 4-5, pp. 51-77.

Pérez, Agustín (2002), Análisis y evaluación de leyes en materia de prevención delictiva, México, FCE/UAM.

Pith, Tamar (2009), “Són posibles les polítiques democràtiques per a la seguretat?”, Revista Catalana de Seguretat Pública maig, pp. 27-46.

Recasens, Amadeu (2003), "Globalización, riesgo y seguridad: el continuóse de que alguien empezóse”, La seguridad en la sociedad del riesgo. Un debate abierto, Barcelona, Atelier, pp. 365-379.

Robert, Philippe (2006), “Seguridad objetiva y seguridad subjetiva”, Revista Catalana de Seguridad Pública, 16, pp. 91-102.

(2003). El ciudadano, el delito y el Estado. Barcelona, Atelier.

Secretaría de Gobernación (s/f), Programa Integral para Prevenir, Atender, Sancionar y Erradicar la Violencia contra las Mujeres, México, SG.

Smart, Carol (1989), Feminism and the power of law, London, Routledge and Kegan Paul.

Sparks, R. (2007), "Perspectivas sobre el riesgo y política penal”, Delito y Sociedad 26(23), pp. 29-47.

Stanko, Elizabeth (2009), “Es pot reduir la por de la delinqüència que tenen les dones?”, Revista Catalana de Seguretat Pública, maig, pp. 47-60.

Unión Europea (2012), Directiva por la que se establecen normas mínimas sobre los derechos, el apoyo y la protección de las víctimas de delitos (directiva 2012/29/UE), Bruselas, UE.

(2010), Programa de Estocolmo 2010-2014. Una Europa abierta y segura que sirva y proteja al ciudadano (doc. 2010/C 115/o1), Bruselas, UE.

(2002), Recomendación [Rec (2002)5], Comité de Ministros del Consejo de Europa. Bruselas, UE. 
Van Swaaningen, René (2011), Perspectivas europeas para una criminología crítica. Argentina, BdeF.

(2005), "La política de seguridad ciudadana en Holanda: Traficando con el miedo”, Revista Española de Investigación Criminológica AC-02-05, pp. 1-21.

(2002), "Hacia un nuevo concepto de seguridad comunitaria", Revista Catalana de Seguridad Pública 11, pp. 221-242.

WARr, M. (1984), “Fear of victimization: Why are women and the elderly more afraid?”, Social Science Quarterly 65, pp. 681-702.

Legislación y programas de gobierno:

Departamento del Distrito Federal (1995), "Programa de Seguridad Pública para el Distrito Federal (PSPDF) 1995-2000”, Diario Oficial de la Federación DVI, 19, primera sección, pp. 24-49.

Gobierno del Distrito Federal (2014), "Programa Sectorial de Seguridad Ciudadana 2013-2018”, Gaceta Oficial del Distrito Federal 1969 bis, pp. 2-98.

(2008), "Programa de Seguridad Pública para el Distrito Federal (PSPDF) 20072012. Seguridad y justicia para la Ciudad de México", Gaceta Oficial del Distrito Federal 250, pp. 130-141.

(2001), "Programa General de Desarrollo del Distrito Federal (PGDDF) 20012006", Gaceta Oficial del Distrito Federal 141, pp. 2-124.

Gobierno del Estado de Guanajuato (2017), Programa Estatal para Prevenir, Atender, Sancionar y Erradicar la Violencia contra las Mujeres, México, IMUG.

(2014), “Decreto no. 191. Ley del Sistema de Seguridad Pública del Estado de Guanajuato”, Periódico Oficial, Gobierno del Estado, Guanajuato, Gto., no. 188.

(2011), "Reglamento de la Ley de Acceso de las Mujeres a una Vida Libre de Violencia para el Estado de Guanajuato", Periódico Oficial, Gobierno del Estado, Guanajuato, Gto., no. 142.

(2010), "Ley de Acceso de las Mujeres a una Vida Libre de Violencia para el Estado de Guanajuato", Periódico Oficial, Gobierno del Estado, Guanajuato, Gto., no. 189.

(2009), "Ley para prevenir, atender y erradicar la violencia en el Estado de Guanajuato”, Periódico Oficial, Gobierno del Estado, Guanajuato, Gto., no. 50.

Secretaría de Gobernación (2014), "Decreto del Programa Integral para Prevenir, Atender, Sancionar y Erradicar la Violencia contra las Mujeres 2014-2018”, Diario Oficial de la Federación DCCXXVII, 25, primera sección-extraordinaria, pp. 2-52.

(2014), “Programa Nacional de Seguridad Pública (PNSP) 2014-2018”, Diario Oficial de la Federación DCCXXVII, 25, cuarta sección 36-101. 
(2014), "Decreto del Programa Nacional para la Prevención Social de la Violencia y la Delincuencia 2014-2018”, Diario Oficial de la Federación DCCXXVII, 25, quinta sección, 1-82.

(2012), "Decreto de la Ley General para la Prevención Social de la Violencia y la Delincuencia”, Diario Oficial de la Federación DCC, 19, primera sección, 2-8.

(2008), "Reglamento de la Ley General de Acceso de las Mujeres a una Vida Libre de Violencia”, Diario Oficial de la Federación DCLIV, 8, primera sección, 2-13.

(2007), "Decreto de la Ley General de Acceso de las Mujeres a una Vida Libre de Violencia”, Diario Oficial de la Federación DCXLI, 1, primera sección, 2-17.

Secretaría de Seguridad Pública (2009), "Programa Nacional de Seguridad Pública (PNSP) 2008-2012", Diario Oficial de la Federación DCLXVI, 18, primera sección, 5-36.

(2003), "Programa Nacional de Seguridad Pública (PNSP) 2001-2006", Diario Oficial de la Federación DXCII, 9, primera sección, 2-48.

Secretaría de Seguridad Pública del Estado de Guanajuato (s.f.), Programa Estatal de Prevención Social. ACTUAR ES PREVENIR, México. 